\title{
Studying dark matter haloes with Schwarzschild modeling
}

\author{
Klaudia Kowalczyk ${ }^{1}$, Ewa L. Łokas $^{1}$ and Monica Valluri ${ }^{2}$ \\ ${ }^{1}$ Nicolaus Copernicus Astronomical Center, Polish Academy of Sciences, \\ Bartycka 18, 00-716 Warsaw, Poland \\ email: klaudia.kowalczyk@gmail.com, lokas@camk.edu.pl \\ ${ }^{2}$ Department of Astronomy, University of Michigan, \\ 1085 South University Ave., Ann Arbor, MI 48109, USA \\ email: mvalluri@umich.edu
}

\begin{abstract}
We present a study exploring the reliability of recovering the mass and velocity anisotropy profiles of dark matter haloes using the Schwarzschild method of modeling galaxies.
\end{abstract}

Keywords. galaxies: dwarf, galaxies: fundamental parameters, galaxies: kinematics and dynamics, Local Group, dark matter

We continued the work first reported in the paper Kowalczyk et al. (2016) focusing on the investigation of the reliability of recovering the profiles of the mass distribution and the anisotropy parameter of numerical realizations of dark matter haloes with the orbitbased modeling method of Schwarzschild (1979). We used four spherically symmetric haloes sharing the same mass profile but differing in the velocity anisotropy. Models of the anisotropy profile include those constant as well as varying with radius that are difficult to study with other methods.

We carried out our research in two steps. First, we integrated a representative library of orbits in the potential generated by the mass distribution matching the one of the haloes, we extracted the observables (the projected mass and the velocity moments) both for the mock data and the orbits from the library and we binned them as a function of radius. We fitted the parameters assuming that the data can be represented as a linear combination of the orbits. We have shown that all anisotropy profiles can be reproduced with a high accuracy for a large sample of particles of the tracer (using all particles from the numerical realizations, i.e. over 200000 ) and similarly well with relatively small errors for a small data sample, corresponding to the sample presently available e.g. for the Fornax dwarf spheroidal galaxy: 100000 positions and 2500 line-of-sight velocities.

In the second part we integrated the orbit libraries for various mass distributions, fitted them to the data and compared the quality of the fits in order to find the best model. We concluded that for the large data sample we were able to recover the true mass profile with the confidence level higher than $5 \sigma$, whereas for the small sample the true profile was located within $1 \sigma$ region regardless of the underlying anisotropy.

The full study will be presented in Kowalczyk, Łokas \& Valluri (2016, in preparation).

\section{References}

Kowalczyk, K., Łokas, E. L., \& Valluri, M. 2016, Proc. of Polish Astronomical Society, in press Schwarzschild, M. 1979, ApJ, 232, 236 